\title{
Alterações cardiovasculares e intracranianas promovidas pela buprenorfina em cães anestesiados com desflurano
}

\author{
Buprenorphine cardiovascular and intracranial alterations in dogs anesthetized with desflurane
}

\author{
Almir Pereira de Souza ${ }^{1}$ Márlis Langenegger de Rezende ${ }^{1}$ Newton Nunes ${ }^{2}$ Celina Tie Nishimori ${ }^{1}$ \\ Paulo Sérgio Patto dos Santos ${ }^{3}$ Danielli Parrilha de Paula ${ }^{1}$
}

RESUMO

\begin{abstract}
Objetivou-se, com a realização deste experimento, estudar possíveis alterações nas variáveis cardiovasculares e intracranianas promovidas pela buprenorfina, em cães anestesiados com desflurano. Para tanto, foram utilizados oito cães adultos, clinicamente saudáveis. A anestesia foi induzida com

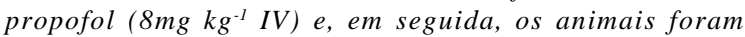
intubados com sonda orotraqueal de Magill, a qual foi conectada ao aparelho de anestesia volátil para administração de desflurano (1,5 CAM). Os animais foram mantidos sob ventilação controlada durante todo o período experimental. Após 20 minutos do posicionamento do cateter de pressão intracraniana (PIC), administrou-se buprenorfina $\left(0,02 \mathrm{mg} \mathrm{kg}^{-1} \mathrm{IV}\right)$. Foram avaliados: PIC; pressão de perfusão cerebral $(P P C) ; F C$; PAS, PAM e PAD; débito cardíaco $(D C)$; pressão venosa central $(P V C) ;$ e pressão da artéria pulmonar (PAP). As colheitas foram feitas nos seguintes momentos: $M 1$ - 20 minutos após o posicionamento do cateter de PIC; M2 - 15 minutos após a administração do opióide; M3, M4 e M5 - de 15 em 15 minutos após M2. A avaliação estatística dos dados foi efetuada por meio de ANOVA seguida do Teste de Tukey $(p<0,05)$. A PIC permaneceu estável durante todo o período experimental. Entretanto, registrou-se uma queda acentuada, estatisticamente significativa, da PPC após o M2. As variáveis cardiovasculares FC, PAS, PAM, PAD, DC e PAP, apresentaram redução significativa de seus valores após $M 2$, mantendo-se estáveis no restante do período experimental. Quanto à PVC, o teste estatístico não registrou alterações significativas. Assim, pôde-se concluir que a buprenorfina não interferiu na PIC. Entretanto, a queda dos índices cardiovasculares, especialmente da PAM, determinada pela administração do opióide, causa redução da PPC em cães anestesiados com desflurano.
\end{abstract}

Palavras-chave: opióides, desflurano, cães, anestesia.

\begin{abstract}
The aim of this work was to study the possible alterations on cardiovascular and intracranial parameters caused by buprenorphine, in dogs anesthetized with desflurane. Eight adult healthy male and female mongrel dogs were used. The anesthetic induction was made using propofol (IV), and immediately after, the dogs were intubated and the tube was connected to a volatile anesthetic circuit, and desflurane was administrated at 1.5 $M A C$. The dogs were intrumented and 20 minutes after the introduction of the intracranial catheter, they received buprenorphine $\left(0.02 \mathrm{mg} \mathrm{kg}^{-1} \mathrm{IV}\right)$. The animals were on mechanical ventilation during all the experimental period. Intracranial pressure (ICP), cerebral perfusion pressure (CPP), Heart Rate (HR); Systolic, Diastolic and Mean Arterial Blood Pressure (SAP, DAP and MAP, respectivelly); Cardiac Output (CO); Central Venous Pressure (CVP) and Pulmonar Arterial Pressure (PAP) were evaluated. The measurements were realized 20 minutes after the introduction of the intracranial catheter (M1); 15 minutes after buprenorphine administration (M2); and at each 15 minutes after M2 (M3, M4 and M5). The numerical data were analysed by use of ANOVA followed by a Tukey's test $(p<0.05)$. Intracranial pressure was stable during all the experimental period, being observed only a decrease of the CPP after buprenorphine administration (M2). After buprenorphine administration (M2), there was a reduction in cardiovascular values: HR, PAP, SAP, DAP and MAP. During all the study, these values remained lower than the initial values, but were within normal values to the species. The CO and CVP were also decreased, but not statistically significant. The results allow us to conclude that buprenorphine didn't interfere with intracranial pressure. Howvever, the marked decrease of arterial pressure due to buprenorphine administration caused a reduction of the cerebral perfusion pressure in dogs anesthetized with desflurane.
\end{abstract}

Key words: opioids, desflurane, dogs, anesthesia

${ }^{1}$ Médico Veterinário, Doutorando em Cirurgia Veterinária, Faculdade de Ciências Agrárias e Veterinárias (FCAV), Universidade Estadual Paulista (UNESP), Campus de Jaboticabal.

${ }^{2}$ Médico Veterinário, Professor, Doutor, Departamento de Clínica e Cirurgia Veterinária, FCAV, UNESP, 14884-900, Jaboticabal,

SP. E-mail: newton@fcav.unesp.br. Autor para correspondência.

${ }^{3}$ Professor de Anestesiologia Veterinária, Centro Universitário Barão de Mauá, Ribeirão Preto, SP. 


\section{INTRODUÇÃO}

Diversos agentes analgésicos opióides têm sido colocados à disposição da classe médica e veterinária. Desta forma, abrem-se possibilidades variadas do uso isolado ou associado destes fármacos aos agentes anestésicos inalatórios da atualidade, obtendo-se assim uma anestesia balanceada de melhor qualidade e maior segurança para os pacientes.

A buprenorfina é um opióide com atividade agonista e antagonista em seres humanos e animais (MARTINEZ et al., 1997). Possui potência analgésica aproximadamente 30 vezes superior a da morfina (THURMON et al., 1996) aliviando a dor moderada a grave, associada a procedimentos cirúrgicos abdominais, torácicos e ortopédicos (GÓRNIAK, 1997). Uma das características hemodinâmicas, conseqüientes da administração deste opióide, em cães, é a redução significativa da freqüência cardíaca, provavelmente por aumento do tono vagal, e alterações mínimas na pressão sanguiínea sistêmica (STEPIEN et al., 1995), apresentando, porém, depressão respiratória que pode ser revertida com o uso de antagonistas opióides, como o naloxone (THURMON et al., 1996).

O desflurano, um anestésico volátil halogenado fluorado, tem peso molecular igual a 168, ponto de ebulição aos $23,5^{\circ} \mathrm{C}$, ao nível do mar, pressão de vapor de $664 \mathrm{mmHg}$, a $20^{\circ} \mathrm{C}$ (SMILEY, 1992), e vem sendo utilizado na rotina cirúrgica desde 1992 (WEISKOPF et al., 1992). O baixo coeficiente sangue/ gás do desflurano $(0,42)$ permite rápido aumento ou diminuição da concentração alveolar, que torna rápida a indução e a recuperação do paciente (EGER, 1992). A administração do desflurano é associada a aumento da atividade simpática, que atinge o pico máximo decorridos 5 minutos de exposição ao fármaco (PACENTINE et al., 1995). A ação simpática deve-se à existência de sítios receptores nas vias aéreas superiores, que respondem rapidamente à elevação da concentração. Esta resposta é resultante da direta ação irritante sobre a mucosa (MUZI et al., 1996).

Porém, foi constatado que o aumento da atividade simpática no homem parece estar relacionada com ativação central e não por irritação das vias aéreas superiores (CIOFOLO \& REIZ, 1999).

No sistema cardiovascular, este anestésico induz queda da pressão arterial média, aumento da frequiência cardíaca (CLARKE et al., 1996), depressão da contratilidade do coração e conseqüente redução do débito cardíaco (PAGEL et al., 1991). O fármaco possui propriedades inibitórias sobre arritmias ventriculares espontâneas, após infarto do miocárdio (NOVALIJAet al., 1998).
No homem, não foram encontradas evidências de que o desflurano induza isquemia do miocárdio ou de que o fármaco aumente a taxa de mortalidade em pacientes com doença coronária (WARLTIER \& PAGEL, 1992). Em coelhos submetidos a infarto experimental, houve marcante diminuição na área afetada e proteção do miocárdio contra injúrias de perfusão (PRECKEL et al., 1998).

A vasodilatação cerebral produzida pelo desflurano deve-se à diminuição dose-dependente da resistência vascular cerebral e ao aumento no fluxo e no volume sangüíneo cerebral (LUTZ et al., 1991; YOUNG \& APFELBAUM, 1995). O aumento do citado fluxo é atenuado com o tempo, indicando que a autorregulação cerebrovascular é preservada. Entretanto pode ocorrer aumento na pressão intracraniana durante o período hábil anestésico (GRAHAM, 1994). O aumento marcante da pressão intracraniana no período trans-anestésico parece resultar em parte do aumento na formação do fluido cérebro-espinhal ou da diminuição da absorção do mesmo (ARTRU, 1993).

Em estudos realizados em pacientes normocapneicos com lesões supratentoriais, em que se comparou desflurano e isoflurano, não foram observadas variações de pressão arterial média, pressão de perfusão cerebral e pressão intracraniana entre os agentes voláteis. Ambos reduziram a pressão arterial média e a pressão de perfusão cerebral, mas a pressão intracraniana permaneceu inalterada (FRAGA et al., 2000).

Objetivou-se, com a realização deste experimento, avaliar as possíveis alterações na dinâmica cardiovascular e nos parâmetros intracranianos decorrentes do uso do opióide buprenorfina em cães, induzidos à plano anestésico com propofol, durante anestesia geral inalatória com desflurano.

\section{MATERIAL E MÉTODO}

Foram utilizados oito cães adultos, cinco machos e três fêmeas, clinicamente saudáveis, com peso de $11,5 \mathrm{~kg}( \pm 2,7)$. Após jejum alimentar de 12 horas e restrição hídrica de duas horas, foi realizada indução à plano anestésico com $8 \mathrm{mg} / \mathrm{kg}$ de propofol ${ }^{\mathrm{a}}$, por via intravenosa (IV) e, em seguida, intubados com sonda de Magill. A sonda foi então coaptada ao aparelho de anestesia inalatória para administração do desflurano, sendo o aparelho de anestesia equipado com ventilador volumétrico ${ }^{\mathrm{b}}$, através de circuito anestésico tipo "semi-fechado"c, dotado de vaporizador termocompensado, microprocessado e 
calibrado para o agente anestésico ${ }^{\mathrm{d}}$, tendo como fluxo diluente o oxigênio $\left(\mathrm{O}_{2}\right.$ a $\left.100 \%\right)$ num fluxo de $30 \mathrm{ml} \mathrm{kg}^{-1} \mathrm{~min}^{-1}$.

O desflurano foi fornecido na concentração de 1,5 CAM, considerando-se a CAM como sendo equivalente a 7,2V\% (CLARKE et al., 1996). O controle da concentração anestésica foi obtido por meio de analisador de gases ${ }^{\mathrm{e}}$, cujo sensor foi colocado em série com o sensor de capnometria e adaptado à extremidade da sonda orotraqueal, conectado ao circuito anestésico.

Ato contínuo, aplicou-se, rocurônio ${ }^{\mathrm{f}}$ na dose de $0,6 \mathrm{mg} \mathrm{kg}^{-1} \mathrm{IV}$. Procedeu-se a ventilação controlada, com amplitude e freqüência suficientes para permitir leitura de capnometria constante em $35 \mathrm{mmHg}$, aferida em oxicapnógrafog ${ }^{\mathrm{g}}$ cujo sensor de fluxo principal foi posicionado na extremidade da sonda orotraqueal conectada ao equipamento de anestesia. $\mathrm{O}$ bloqueio neuromuscular foi mantido durante todo o período experimental através de infusão contínua de rocurônio $\left(0,6 \mathrm{mg} \mathrm{kg}^{-1} \mathrm{~h}^{-1}\right)$. Os animais foram instrumentados e 20 minutos após o posicionamento do cateter de pressão intracraniana, foi administrada a buprenorfina ${ }^{\mathrm{h}}\left(0,02 \mathrm{mg} \mathrm{kg}^{-1} \mathrm{IV}\right)$. Durante toda a fase experimental a temperatura foi mantida estável pelo uso de colchão térmicoi.

Foram avaliados: a pressão intracraniana (PIC), através de mensuração digital direta em monitor de pressão intracraniana ${ }^{j}$, cujo cateter de fibra óptica ${ }^{k}$ foi implantado cirurgicamente, com o uso de "kit" de acesso $^{1}$, na superfície do córtex cerebral direito, segundo técnica descrita por BAGLEY et al. (1995); pressão de perfusão craniana (PPC), obtida pela diferença entre a pressão arterial média e a PIC; freqüência cardíaca (FC), obtida em monitor de eletrocardiografia computadorizado ${ }^{\mathrm{m}}$, pelo cálculo do intervalo R-R; pressões arteriais sistólica, média e diastólica (PAS, PAM e PAD, respectivamente), obtidas pela cateterização da artéria femoral esquerda ou direita, cuja leitura foi feita em monitor digital computadorizadon $^{\text {; }}$ débito cardíaco (DC), obtido após introdução do cateter de Swan-Ganz pela veia femoral (esquerda ou direita) até o correto posicionamento na artéria pulmonar, cuja leitura foi obtida através do mesmo monitor utilizado para pressão arterial; pressão venosa central (PVC) e pressão da artéria pulmonar (PAP), ambas obtidas pelo mesmo cateter utilizado para o DC.

As determinações foram feitas nos seguintes momentos: M1 - 20 minutos após o implante do cateter da PIC; M2 - 15 minutos após a administração do opióide; M3, M4 e M5 - de 15 em 15 minutos após M2. A avaliação estatística dos dados foi efetuada por meio de Análise de Variância (ANOVA), seguida do Teste de Tukey $(p<0,05)$.

\section{RESULTADOS}

Pôde-se observar que, após a administração da buprenorfina (M2), os parâmetros cardiovasculares apresentaram redução significativa, com exceção do DC e PVC, pelo teste estatístico empregado. Durante todo o período experimental estes valores permaneceram abaixo dos registrados inicialmente, porém dentro dos limites considerados fisiológicos para a espécie.

Em relação à FC (Tabela 1), pode-se observar um acentuado decréscimo de seus valores após M2 $(91,38 \pm 10,01)$, quando comparado a seus índices iniciais $(M 1=118,25 \pm 12,13)$. Houve uma estabilidade dos dados após a queda registrada inicialmente (M2 à M5). O mesmo comportamento de queda pode ser observado quando se analisa os dados referentes às pressões arteriais (Tabela 1) com o uso apenas do desflurano, ou seja M1 (PAS = 125,38+12,13; $\mathrm{PAD}=71,13 \pm 14,53$; $\mathrm{PAM}=92,00 \pm 14,64$; e PAP $=$ $16,25 \pm 1,49)$ para aqueles a partir de $\mathrm{M} 2(\mathrm{PAS}=110,63$ $\pm 11,75 ; \mathrm{PAD}=57,63 \pm 8,33 ; \mathrm{PAM}=77,00 \pm 10,11 ; \mathrm{e}$ $\mathrm{PAP}=14,63 \pm 1,41)$. A PAD continuou a manifestar tendências a queda, embora o teste estatístico não tenha detectado diferenças ao longo dos momentos, após M2. O DC e a PVC foram as outras variáveis cardiovasculares que seguiram a mesma tendência de redução registrada nas variáveis citadas anteriormente (Tabela 1). Houve, de modo semelhante ao ocorrido com as variáveis anteriores, estabilização dos valores, não apresentando alteração estatisticamente significativa. $\mathrm{O}$ mesmo resultado foi observado com relação à PAP (M1 = 16,25+1,49), que reduziu a partir da administração da buprenorfina (M2 = 14,63+1,41).

A PIC (Tabela 1) permaneceu estável durante todo o período experimental. Foi observada uma discreta redução da pressão (de 19,50 $\mathrm{mmHg}+5,68$ em M1 para 16,13 mmHg $\pm 3,48$ em M2) após a administração do opióide, não diferindo, entretanto, pela análise estatística empregada. Da mesma forma registrou-se uma redução estatisticamente significativa da PPC (M1 = 72,50 $\pm 13,53)$ comparado ao momento seguinte à aplicação da buprenorfina $(60,88$ $\mathrm{mmHg} \pm 11,14)$, que se manteve ao longo de todo o período experimental.

\section{DISCUSSÃO}

Embora não se tenha utilizado um grupo de animais recebendo desflurano, acrescido de placebo, 
Tabela 1 - Valores médios e desvios padrão (entre parênteses) das variáveis cardiovasculares e intracranianas de cães medicados com buprenorfina durante anestesia com desflurano.

\begin{tabular}{|c|c|c|c|c|c|}
\hline \multirow{2}{*}{ Variável } & \multicolumn{5}{|c|}{ Momentos } \\
\hline & M1 & M2 & M3 & M4 & M5 \\
\hline \multirow{2}{*}{ FC (bat./min.) } & $118,25 \mathrm{a}$ & $91,38 b$ & $94,25 b$ & $96,63 b$ & $94,75 b$ \\
\hline & $(12,13)$ & $(10,01)$ & $(9,59)$ & $(9,74)$ & $(11,50)$ \\
\hline \multirow{2}{*}{ PAS (mmHg) } & $125,38 \mathrm{a}$ & $110,63 b$ & $108,75 b$ & $110,75 b$ & $107,00 \mathrm{~b}$ \\
\hline & $(14,88)$ & $(11,75)$ & $(11,90)$ & $(13,35)$ & $(9,78)$ \\
\hline \multirow{2}{*}{ PAD (mmHg) } & $71,13 \mathrm{a}$ & $57,63 \mathrm{~b}$ & $56,88 \mathrm{~b}$ & $56,88 \mathrm{~b}$ & $55,00 \mathrm{~b}$ \\
\hline & $(14,53)$ & $(8,33)$ & $(8,29)$ & $(8,85)$ & $(6,12)$ \\
\hline \multirow{2}{*}{ PAM (mmHg) } & $92,00 \mathrm{a}$ & $77,00 \mathrm{~b}$ & $76,63 b$ & $77,73 b$ & $74,75 b$ \\
\hline & $(14,64)$ & $(10,11)$ & $(9,98)$ & $(10,65)$ & $(8,17)$ \\
\hline \multirow{2}{*}{ DC (L/min.) } & $2,06 \mathrm{a}$ & $1,84 \mathrm{~b}$ & $1,96 \mathrm{~b}$ & $1,93 b$ & $1,85 \mathrm{~b}$ \\
\hline & $(0,38)$ & $(0,34)$ & $(0,46)$ & $(0,35)$ & $(0,24)$ \\
\hline \multirow{2}{*}{ PVC (mmHg) } & 4,25 & 4,00 & 4,13 & 3,50 & 3,75 \\
\hline & $(1,28)$ & $(1,51)$ & $(1,46)$ & $(1,20)$ & $(1,39)$ \\
\hline \multirow{2}{*}{ PAP (mmHg) } & $16,25 \mathrm{a}$ & $14,63 b$ & $14,13 b$ & $15,00 \mathrm{~b}$ & $14,63 b$ \\
\hline & $(1,49)$ & $(1,41)$ & $(1,81)$ & $(1,77)$ & $(1,60)$ \\
\hline \multirow{2}{*}{ PIC (mmHg) } & 19,50 & 16,13 & 16,88 & 17,50 & 17,75 \\
\hline & $(5,68)$ & $(3,48)$ & $(4,29)$ & $(4,60)$ & $(5,95)$ \\
\hline \multirow{2}{*}{ PPC (mmHg) } & $72,50 \mathrm{a}$ & $60,88 \mathrm{~b}$ & $59,75 \mathrm{~b}$ & $60,13 b$ & $57,00 \mathrm{~b}$ \\
\hline & $(13,53)$ & $(11,14)$ & $(10,54)$ & $(11,29)$ & $(8,80)$ \\
\hline
\end{tabular}

Para cada variável, médias seguidas pela mesma letra na linha, não diferem significativamente pelo teste de Tukey ( $\mathrm{p}<0,05$ ).

FC - freqüência cardíaca; PAS, PAM e PAD - pressão arterial sistólica, média e diastólica, respectivamente; DC - débito cardíaco; PVC pressão venosa central; PAP - pressão da artéria pulmonar; PIC - pressão intracraniana; PPC - pressão de perfusão cerebral

de modo a simular a administração de buprenorfina, a metodologia pôde ser considerada adequada, para que se atingissem os objetivos, uma vez que se protocolou o método conforme sugerido por outros estudiosos (GREENE et al., 1990; GROSS et al., 1993). Os autores basearam as mensurações em teste comparativo entre vários momentos e aquele inicial, quando os pacientes haviam recebido apenas o anestésico volátil. A repetição deste método forneceu valores considerados confiáveis e permitiu o cotejamento das variáveis entre os momentos, permitindo reduzir o número de animais, o que é desejável, do ponto de vista ético e minimizando os custos do experimento.

Optou-se por realizar o experimento em condições de normocapnia, com o emprego de ventilação controlada, mediante o uso de bloqueio neuromuscular periférico. O miorrelaxante empregado não é dotado de características que possam interferir sobre as variáveis intracranianas (PIC e PPC) e pressão arterial média (SCHRAMM et al., 1996). Sendo assim, é possível especular quanto a possíveis interferências da buprenorfina sobre a ventilação espontânea, o que poderia levar ao aumento dos níveis arteriais de dióxido de carbono, com conseqüente elevação da PIC, caso o opióide determinasse redução na freqüência ou amplitude da respiração (THURMON et al., 1996). Desta forma, os efeitos da associação entre o desflurano e a buprenorfina, em pacientes sob ventilação espontânea e sua influência em variáveis intracranianas merece investigações complementares.

No tocante aos parâmetros cardiovasculares, pôde-se observar que estes estavam dentro de níveis considerados fisiológicos para a espécie estudada, antes da aplicação do agente opióide. Tanto a FC como as pressões sistólica, diastólica, média, e da artéria pulmonar, não manifestaram intensamente as ações simpatomiméticas típicas do desflurano (CLARKE et al., 1996). Tal estabilidade cardiovascular resultou em manutenção do débito cardíaco e da pressão venosa central, não sendo registradas reduções dignas de nota, conforme o encontrado na literatura (PAGEL et al., 1991).

A queda da FC observada após a administração da buprenorfina (M2) demonstra sua ação cronotrópica negativa, podendo ser proveniente de efeito vagotônico central (STEPIEN et al., 1995). No tocante a pressão arterial, pôde-se notar que não ocorreram alterações clínicas marcantes ao longo do período anestésico, embora a partir de M2 as variações terem sido consideradas significativas pelo teste 
estatístico empregado. Porém, é interessante ressaltar que a PAD foi a que teve queda mais acentuada, a qual poderia estar diretamente relacionada à diminuição da resistência vascular periférica (GANONG, 1993). A mesma explicação pode ser atribuída à PAP, em que se evidenciou declínio do seu índice inicial, após a administração do opióide.

Em relação ao DC, o uso da buprenorfina determinou uma redução significativa dos valores. Tal achado foi obtido em função da redução da FC provocada pelo opióide (STEPIEN et al., 1995), bem como por uma provável redução da força contrátil do miocárdio.

Pôde-se constatar que a PVC seguiu a tendência manifestada nas demais variáveis cardiovasculares, ou seja, queda após a administração da buprenorfina, notoriamente mais intensa aos 45 minutos após a sua aplicação. Este efeito pode estar relacionado à diminuição do DC, promovida pelo desflurano (PAGEL et al., 1991), associado à diminuição da FC e da pressão arterial sistêmica, promovidas pela buprenorfina (STEPIEN et al., 1995), com eventual redução na pré-carga.

A pressão intracraniana (PIC) permaneceu, durante toda a fase experimental, acima do valor máximo, citado na literatura, de $15 \mathrm{mmHg}$ (SHORT \& OTTO, 1996). Esta elevação estaria intrinsecamente relacionada a alterações na pressão do fluido cérebroespinhal, oriunda de uma elevação da atividade simpática, característica do agente anestésico inalatório empregado (GRAHAM, 1994; CIOFOLO \& REIZ, 1999).

Não foi possível constatar neste estudo, durante o uso isolado do desflurano (M1), redução da pressão de perfusão cerebral (FRAGA et al., 2000), haja vista esta variável depender diretamente da PAM que permaneceu discretamente elevada. Entretanto, a partir da adição da buprenorfina ao protocolo anestésico (M2), pôde-se observar redução tanto da PIC como da PPC, sendo que apenas esta última teve significado estatístico. A literatura é carente de informações acerca da ação deste fármaco opióide em parâmetros intracranianos. Assim, com os dados obtidos neste estudo, pode-se inferir uma ausência de efeitos diretos na PIC e presença de efeitos indiretos na PPC, a qual foi reduzida certamente por uma ação do opióide na PAM, conforme discutido anteriormente.

Assim, com base nos resultados obtidos com esta metodologia empregada, pode-se concluir que a buprenorfina promove reduções nos índices cardiovasculares de cães durante anestesia com desflurano determinando, desta maneira, redução na pressão de perfusão cerebral. Outrossim, pode-se admitir como hipótese conclusiva que este opióide não interfere na pressão intracraniana de cães anestesiados com desflurano.

\section{AGRADECIMENTO}

Os autores agradecem à Fundação de Amparo à Pesquisa no Estado de São Paulo (FAPESP) pelo apoio financeiro concedido.

\section{FONTEDEAQUISIÇÃO}

aPROVIVE - Zeus Lifesciences Ltda., São Paulo - SP. bOHMEDA - mod. 7900 - Datex Ohmeda, Miami - EUA ${ }^{\mathrm{c}}$ OHMEDA - mod. Excel 210SE - Datex Ohmeda, Miami EUA

dOHMEDA - mod. TEC 6 - Datex Ohmeda, Miami - EUA 'OHMEDA - mod. 5220 - Datex Ohmeda, Miami - EUA ${ }^{\mathrm{f}}$ ESMERON - ORGANON TEKNIKA B.V./AKZO NOBEL Ltda., São Paulo - SP

gDIXTAL - mod. CO SMO 7100 - DIXTAL Biomédica Ind. Com. Ltda., São Pauló - SP

hTEMGESIC - Schering-Plough S.A., Rio de Janeiro - RJ.

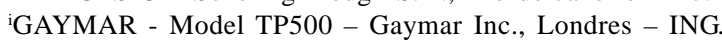
${ }^{\mathrm{j} C A M I N O}$ LABS - mod. MPM - 1 - NeuroCare Groups, San Diego , Califórnia - USA

${ }^{k}$ CAMINO LABS - mod. 110 - 4BT - NeuroCare Groups, San Diego , Califórnia - USA

${ }^{\text {I}}$ CAMINO LABS - mod. 5H-ITH - NeuroCare Groups, San Diego , Califórnia - USA

mTEB - Model ECGPC 1.10 - Tecnologia Eletrônica Brasileira, São Paulo - SP

nDIXTAL DX 2010 - DIXTAL Biomédica Ind. Com. Ltda., São Paulo - SP

\section{REFERÊNCIAS BIBLIOGRÁFICAS}

ARTRU, A.A. Rate of cerebrospinal fluid formation, resistance to reabsorption of cerebrospinal fluid, brain tissue water content, and electroencephalogram during desflurane anestesia in dogs. J Neurosurg Anesthesiol, v.5, n.3, p.178-86, 1993

BAGLEY, R.S. et al. Pathologic effects in brain after intracranial pressure monitoring in clinically normal dogs, using a fiberoptic monitoring system. Am J Vet Res, v.56, n.11, p.1475-1478, 1995.

CIOFOLO, M.J.; REIZ, S. Circulatory effects of volatile anesthetic agents. Minerva Anestesiol, v.65, n.5, p.232$238,1999$.

CLARKE, K.W. et al. Cardiopulmonary effects of desflurane in the dog during spontaneous and artificial ventilation. Res Vet Sci, v.61, p.82-86, 1996.

EGER, E.I II. Desflurane animal and human pharmacology: aspects of kinetics, safety, and MAC. Anesth Analg, v.75, p.3-9, 1992.

FRAGA, M. et al. Intracranial pressure in patients with supratentorial lesions: comparison of effects of desflurane and isoflurane. Eur J Anaesthesiol, v.17, n.19, p.93-99, 2000 .

Ciência Rural, v.34, n.3, mai-jun, 2004. 
GANONG, W.F. Dinâmica do sangue e do fluxo linfático. In: Fisiologia médica. Rio de Janeiro : Prentice-Hall do Brasil, 1993. p.408-420.

GÓRNIAK, S.L. Hipnoanalgésicos e neuroleptoanalgesia. In: SPINOSA, H.S.; BERNARDI, M.M.; GÓRNIAK, S.L. (Eds). Farmacologia aplicada à medicina veterinária. Rio de Janeiro: Guanabara Koogan, 1997. Cap.15. p.140-146.

GRAHAM, S.G. New drug in volatile anaesthesia - desflurane. Ann Acad Med Singapore, v.23, n.4, p.510-518, 1994.

GREENE, S.A.; HARTSFIELD, S.M.; TYNER, C.L. Cardiovascular effects of butorphanol in halothaneanesthetized dogs. Am J Vet Res, v.51, n.8, p.1276-1279, 1990.

GROSS, M.E.; SMITH, J.A.; TRANQUILLI, W.J. Cardiorespiratory effects of combined midazolam and butorphanol in isoflurane-anesthetized cats. Vet Surg, v.22, n.2, p.159-162, 1993.

LUTZ, L.J.; MILDE, J.H.; MILDE, L.N. The response of the canine cerebral circulation to hyperventilation during anesthesia with desflurane. Anesthesiology, v.74, n.3, p.504507, 1991.

MARTINEZ, E.A. et al. Cardiovascular effects of buprenorphine in anesthetized dogs. Am J Vet Res, v.58, n.11, p.1280-1283, 1997.

MUZI, M. et al. Site(s) mediating sympathetic activation with desflurane. Anesthesiology, v.85, n.4, p.737-747, 1996.

NOVALIJA, E. et al. Effects of desflurane, sevoflurane and halothane on postinfarction spontaneous dysrhythmias in dogs. Acta Anaesthesiol Scand, v.42, n.3, p.353357,1998 .

PACENTINE, G.G.; MUZI, M.; EBERT, T.J. Effects of fentanyl on sympathetic activation associated with the administration of desflurane. Anesthesiology, v.82, p.823$831,1995$.
PAGEL, P.S. et al. Influence of volatile anesthetics on myocardial contractily in vivo: desflurane versus isoflurane. Anesthesiology, v.74, p.900-907, 1991.

PRECKEL, B. et al. Effects of enflurane, isoflurane, sevoflurane and desflurane on reperfusion injury after regional myocardial ischaemia in the rabbit heart in vivo. Br J Anaesth, v.81, n.6, p.905-912, 1998.

SCHRAMM, W.M. et al. Effects of rocuronium and vecuronium on intracranial pressure, mean arterial pressure and heart rate in neurosurgical patients. Br J Anaesth, v.77, n.5, p.607-611, 1996.

SHORT, C.E.; OTTO, K.A. The central nervous system. In: THURMON, J.C.; TRANQUILLI, W.J.; BENSON, G.J. (Eds). Lumb \& Jones' veterinary anesthesia. 3.ed. Philadelphia: Lea \& Feabiger, 1996. Chapt.7A, p.148-170.

SMILEY, R.M. An overview of induction and emergence characterictics of desflurane in pediatric, adult, and geriatric patients. Anesth Analg, v.75, p.38-46, 1992.

STEPIEN, R.L. et al. Cardiorespiratory effects of acepromazine maleate and buprenorphine hydrochloride in clinically normal dogs. Am J Vet Res, v.56, n.1, p.78-84, 1995.

THURMON, J.C.; TRANQUILLI, W.J.; BENSON, G.J. Injectable anesthetics. In:______. Lumb \& Jones' veterinary anesthesia. 3.ed. Philadelphia : Lea \& Feabiger, 1996. Chapt.9. p.210-240.

WARLTIER, D.C.; PAGEL, P.S. Cardiovascular and respiratory actions of desflurane: is desflurane different from isoflurane? Anesth Analg, v.75, p.17-31, 1992.

WEISKOPF, R.B. et al. Desflurane does not produce hepatic or renal injury in human volunteers. Anesth Analg, v.74, p.570574,1992 .

YOUNG, C.J.; APFELBAUM, J.L. Inhalational anesthetics: desflurane and sevoflurane. J Clin Anesth, v.7, n.7, p.564577, 1995. 